\title{
Online Monitoring System Design of Intelligent Circuit Breaker Based on DSP and ARM
}

\author{
Meng Song ${ }^{1}$ \\ College of Electronic and Electrical Engineering \\ Shanghai University of Engineering Science \\ Songjiang District, Shanghai 201620, China \\ Yuchen Chen $^{3}$ \\ College of Electronic and Electrical Engineering \\ Shanghai University of Engineering Science \\ Songjiang District, Shanghai 201620, China
}

\author{
Liping Zhang ${ }^{2 *}$ \\ College of Electronic and Electrical Engineering \\ Shanghai University of Engineering Science \\ Songjiang District, Shanghai 201620, China \\ Weijin Zheng ${ }^{4}$ \\ College of Electronic and Electrical Engineering \\ Shanghai University of Engineering Science \\ Songjiang District, Shanghai 201620, China
}

\begin{abstract}
In order to accurately analyze the dynamic characteristics of the vacuum circuit breaker, a dual-core master-slave processor structure for online monitoring system based on DSP and ARM is proposed. This structure consists of host computer, lower computer and signal processing modules. The lower computer uses DSP as the core, which completes acquisition and data preprocessing of circuit breaker's dynamic characteristics through sensors and signal conditioning circuits. The host computer uses ARM as the core which is responsible for task management, analysis, processing and displaying collected data via Ethernet. The communication between DSP and ARM is conducted by HPI. This design improves the reliability of intelligent control unit for the circuit breaker. The experiment showed that this system works steadily and accuracy.
\end{abstract}

Keywords—circuit breaker; online monitoring; ARM; DSP

\section{INTRODUCTION}

The vacuum circuit breakers is not only the most important control and protection equipment in power system, but also the equipment with most frequent mechanical and electrical operation in substations, which affects the stability and reliability of the power grid directly[1]. How to implement online monitoring of vacuum circuit breakers more efficiently has become an important technical topic for domestic and foreign engineers. With the popularization of smart grid technology, the power system is developing to intelligent control system. As an important part of the grid, the breaker's intelligent level has a significant impact on intelligent power grids. The embedded monitoring system in circuit breakers for online intelligent monitoring has become a major research topic for intelligent circuit breaker [2 4].

Online condition monitoring is the basis for the condition maintenance. Existing online monitoring system has the following problems: less monitoring parameter and single function. There are still some deficiencies in management and exchange of the data, scalability and remote maintenance in the system. Moreover, it is difficult to make a systematic and comprehensive evaluation in the system. In order to overcome these deficiencies mentioned above, a dual-core master-slave processor structure for online monitoring system based on
ARM and DSP is proposed. ARM is typical of low power consumption, rich interfaces and strong ability to control, and DSP process data fast with high accuracy. The combination with ARM and DSP will improve the real-time and versatility of the circuit breaker online monitoring system.

\section{SySTEM STRUCTURE}

The intelligent vacuum circuit breaker online monitoring system is composed by the host computer, the lower computer and signal processing modules. The lower computer hardware platform consists of a TMS320F2812 DSP and peripheral hardware circuits. It collects mechanical parameters, divideshut brake circuit current signal and vibration signal of vacuum circuit breaker. The host computer uses ARM as a core, mainly working as remote communication with the host computer, and getting the results of data processing and eigenvalues from the DSP at the same time. The preprocessed data is transferred from DSP to ARM via a HPI interface, and transmitted via the Ethernet interface to the host computer for data analysis and processing, so as to determine the current status of the circuit breaker, and analyze its operation situation and diagnose malfunctions. The system structure is shown as Fig1.

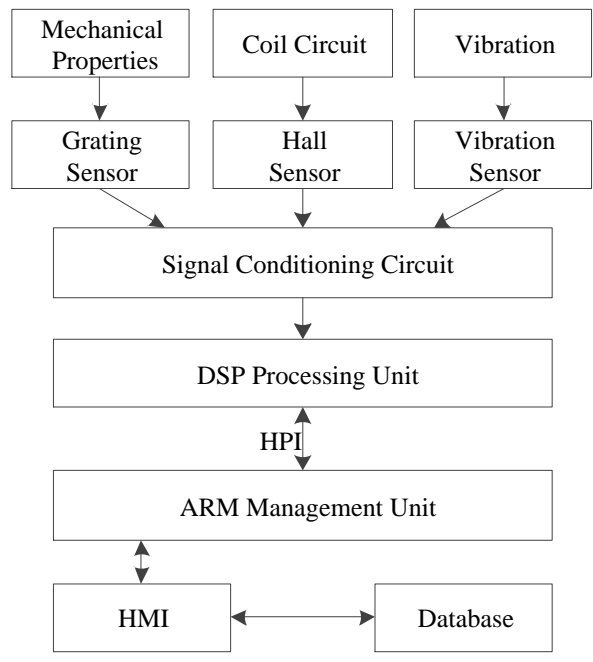

Fig. 1. The Online Monitoring System Diagram 


\section{THE EXTRACTION OF ONLINE MONITORING CHARACTERISTIC QUANTITY}

Main monitoring items for vacuum circuit breaker include: mechanical characteristics, divide-shut brake circuit signal and vibration signal.

Main monitoring items of the mechanical properties of the vacuum breaker include the time, speed, distance and overtravel of the divide-shut brake [5]. The connection between contacts and actuator of circuit breaker is shown as Fig2. Among them, 1 is the moving contact, 2 is the static contact, the length of the link rod 3 , link rod 4 , link rod 5 are $L_{1}, L_{2}, L_{3}$ respectively.

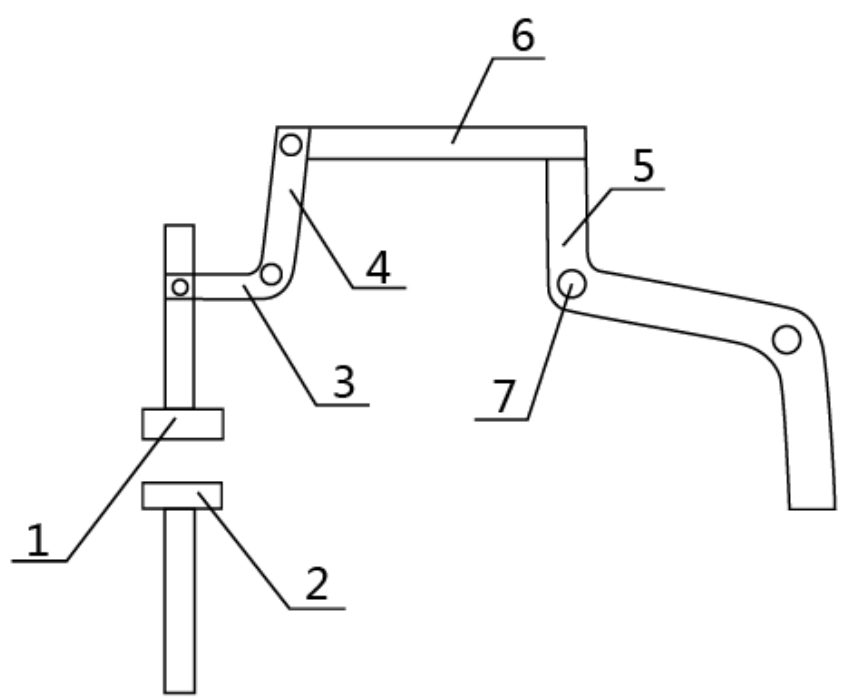

Fig. 2. The Structure for Connection of Contact and Actuator

Due to the structure is very compact and with high potential isolation problem, sensor can't be installed in the insulated rod of moving contact. Thus, the system is installed the angular displacement sensor on the actuator spindle of circuit breaker. According to the relationship between the measured voltage change amount $\Delta \mathrm{U}$ and the reference voltage $U_{0}$, angular displacement sensor turned as the angle $\alpha$ and measured spindle turned as the angle $\beta$ with the following relationship:

$$
\beta=\alpha \cdot \Delta U / U_{0}
$$

The actuator spindle turned the angle as $\beta$, the displacement of insulated pillar linear is $\mathrm{X}$, the link rod 3, 4 turned angle as $\gamma$ and the displacement of dynamic contact linear $\mathrm{Y}$ has the following relationship:

$$
\begin{gathered}
L_{3}{ }^{2}-X^{2}=2 \cos \gamma \cdot L_{3}^{2} \\
L_{2}{ }^{2}-X^{2}=2 \cos \gamma \cdot L_{2}^{2} \\
L_{1}^{2}-Y^{2}=2 \cos \gamma \cdot L_{1}^{2} \\
Y=\left(L_{1} \cdot L_{3} / L_{2}\right) * \sqrt{1-2 \cos \beta}
\end{gathered}
$$

Meanwhile, the test system reads the pulse signal with a sampling frequency and obtains the trip - time characteristic curve of the circuit breaker during the operation. Using the disposed principal axis angular displacement - time curve of the divide-shut brake we can obtain the linear displacement - time curve for the moving contact, and then obtain the time, velocity, distance and overtravel of the divide-shut brake [6].

The main monitoring items of the divide-shut brake circuit include divide-shut brake coil current, the current and voltage of charging motor. Since the judgment is based on whether the current pass through the coil of divide-shut brake or not, the measure of coil currents has direct impact on the measurement accuracy and stability of system. Therefore, hall sensor with high precision, good linearity, small size and good dynamic performance is used to complete data acquisition.

The vibration signal, which consists of a series of transient waveforms, contains a large number of status information of device. Each transient waveform is reflected by the "incident" signal during breaker operation. Vibration is a response to the internal excitation source of the device, identifying the vibration excitation source through appropriate means of detection and signal processing methods so as to find out the source of the fault.

\section{HARDWARE DESIGN}

The hardware platform of the dual-core master-slave processor online monitoring system for vacuum circuit breaker consists of ARM, DSP and signal conditioning circuit. The main processor consists of ARM and peripheral circuits, works as embedded operating system between the user and the coprocessor. ARM manages and maintains the system configuration and communicates with the host computer via Ethernet. At the same time, ARM accesses the processing data and calculated eigenvalues from DSP in real-time.

As the coprocessor of lower computer, DSP is divided into three parts in accordance with the function: ARM interface circuit, DSP coprocessor circuits and power systems. ARM interface circuitry is used to expand peripheral interface circuit of ARM core board, including UART, USB, Ethernet. The lower computer uses TMS320F2812 DSP as core, and uses host interface HPI port as master-slave processor synchronization and communications hardware interface. ARM completes the management task for the lower computer, and power system supply power for these two chip modules.

\section{A. Interface Design}

Communication between ARM and DSP is performed by HPI (Host Port Interface). HPI is the chip peripheral to communicate with the host computer. The host computer can easily access all the address space of DSP and control it via HPI. Compared to the dual-port RAM, serial interface, HPI does not need to add peripheral logic circuits. There is not extra hardware and software overhead in communication with the host computer via HPI. DSP doesn't interrupt normal program running because it can coordinate with hardware conflict itself. HPI is suitable for the applications of high real-time requirements, a large quantity of data rate $[7,8]$.

TMS320F2812 is connected with ARM by the separate 16bit data lines HD and nine control lines as shown in Fig 3. In the process of communication between ARM and DSP via HPI, DSP only needs active participation in interrupting state. DSP is in a passive state in other state. DSP is equivalent to an external memory for ARM. 


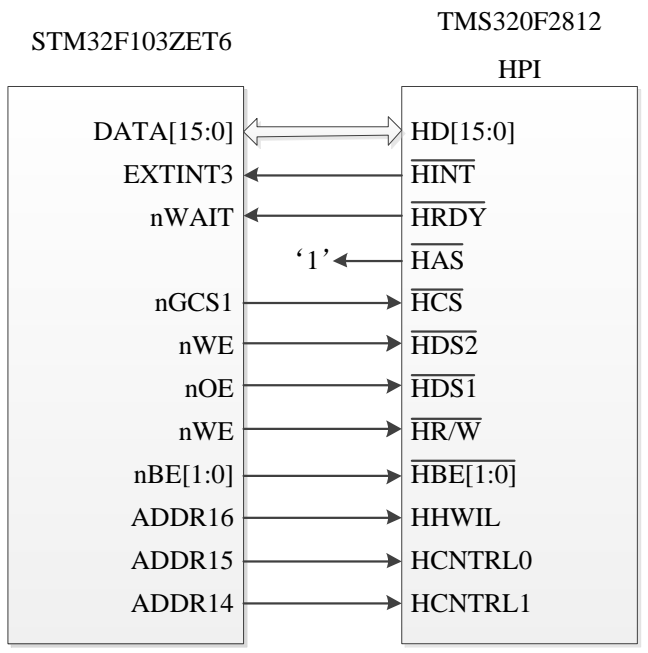

Fig. 3. Hardware Interface

\section{B. Program Development}

Firstly, the DSP firmware is divided by the according functions and required to complete initialization, data acquisition, data processing, data storage, which correspond to three task threads, defined as TskSplit, TskProcess and TskSave. Data acquisition is to obtain real-time data from the sensor, and then the data is divided by channel. The data processing mainly is to process real-time data by channel. There may have one or more arithmetic unit within the channel depending on the application needs, by which to analysis data and obtain the status information of the object. Data storage is to storage processed data to the specified physical address space according to the agreed format.

The processes for the entire program are:

Firstly, the device driver notifies TskSplit to access data from the object data via SIO after obtaining data from the external device. TskSplit will be used after data is read and divided into channel data zones.

Secondly, TskProcess executes the processing program by channel sequentially, and stores the processed data to the processing data buffer.

Finally, TskSave gets data through the pointer of data buffer to organize and store, then returns the empty data buffer after be read to TskProcess.

\section{SOFTWARE DESIGN}

The system software coordinates with hardware to achieve the monitoring of the circuit breaker's dynamic characteristics. The program flow is shown as Fig 4.

The software system's task is to complete initialization of the system, extraction of the signal, data processing, information monitoring and communications with the host computer. Firstly, the program initializes each module, and opens the interrupt event after the system is powered.
When vacuum circuit breaker is opened and closed, an A/D channel starts to collect the opening and closing current signal, the vibration signal parameters and mechanical properties by the way of interruption. Then the data processing program performs. Finally, the data is transferred to the liquid crystal display module. If the lower computer has action, the host computer receives data recovery, then the host computer call data from the lower computer.

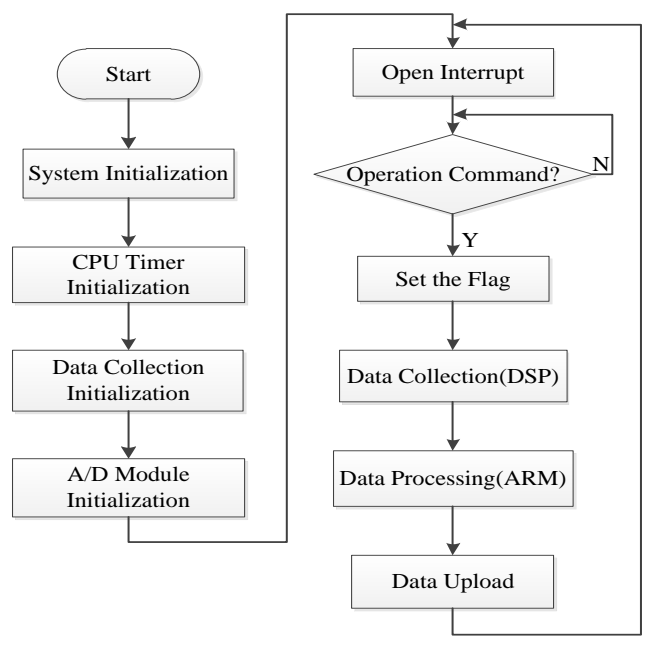

Fig. 4. Software Program Flow Chart

\section{Test Result}

The online monitoring system device is installed on ZN63A-12 vacuum circuit breaker. The parameters of the coil circuit signal and mechanical properties are tested. The results of the coil circuit shown in Table I is the comparison between oscilloscope and experimental data.

TABLE I. Circuit Electrical PARAmEters Results

\begin{tabular}{ccccc}
\hline Parameter & $\begin{array}{c}\text { Control } \\
\text { Voltage(V) }\end{array}$ & $\begin{array}{c}\text { Closing } \\
\text { Current(A) }\end{array}$ & $\begin{array}{c}\text { Tripping } \\
\text { Current(A) }\end{array}$ & $\begin{array}{c}\text { Storage } \\
\text { Current(A) }\end{array}$ \\
\hline Oscilloscope & 113.25 & 1.97 & 1.93 & 2.01 \\
System & 114.03 & 2.01 & 1.96 & 1.98 \\
Error & $0.69 \%$ & $2 \%$ & $1.6 \%$ & $1.5 \%$ \\
\hline
\end{tabular}

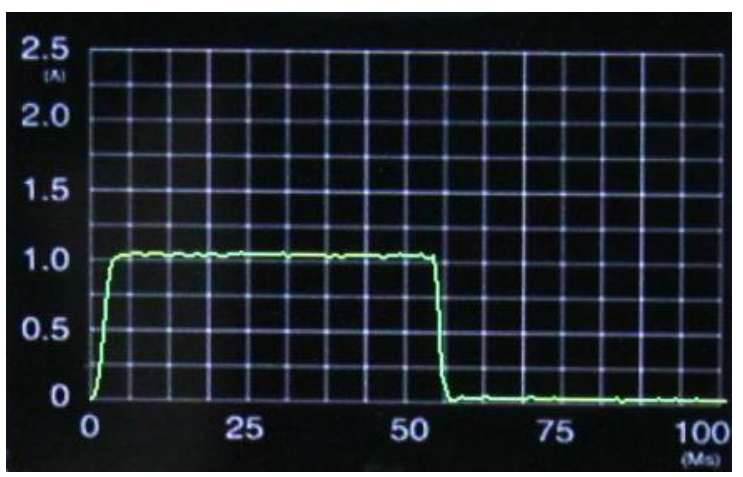

Fig. 5. The Current Curve of Coil Circuit 
The current curve of coil circuit is shown as Fig 5. The result shows that the error between the system values and standard values is small.

In order to test the mechanical properties of the circuit breaker, the monitoring system is compared with mechanical properties tester.

The results are shown in Table II and the tripping displacement - time characteristic curve and closing displacement - time characteristic curve are shown as Fig 6 and Fig 7.

The result shows that the error between the system values and standard values is small.

TABLE II. THE TEST RESUlts OF MECHANICAL PROPERTIES

\begin{tabular}{ccccc}
\hline Parameter & $\begin{array}{c}\text { Distance } \\
(\mathrm{mm})\end{array}$ & $\begin{array}{c}\text { Closing } \\
\text { Time }(\mathrm{ms})\end{array}$ & $\begin{array}{c}\text { Closing } \\
\text { Speed(m/s) }\end{array}$ & $\begin{array}{c}\text { Tripping } \\
\text { Time }(\mathrm{ms})\end{array}$ \\
\hline Tester & 11.879 & 32.246 & 0.79 & 22.03 \\
System & 11.537 & 33.014 & 0.81 & 21.74 \\
Error & $2.9 \%$ & $2.4 \%$ & $2.5 \%$ & $1.3 \%$ \\
\hline
\end{tabular}

\begin{tabular}{ccc}
\hline Parameter & $\begin{array}{c}\text { Tripping } \\
\text { Speed }(\mathrm{m} / \mathrm{s})\end{array}$ & $\begin{array}{c}\text { Ultra-trip } \\
(\mathrm{mm})\end{array}$ \\
\hline Tester & 0.98 & 2.97 \\
System & 0.965 & 3.08 \\
Error & $1.5 \%$ & $3.7 \%$ \\
\hline
\end{tabular}

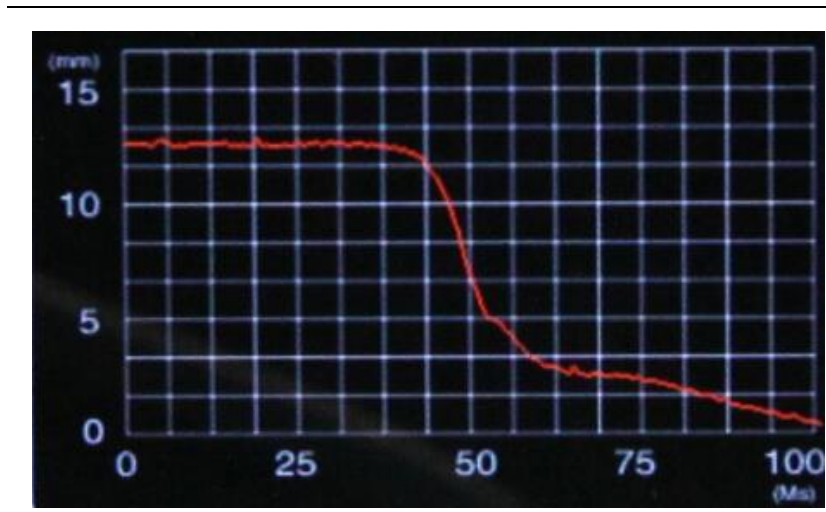

Fig. 6. Tripping Displacement - Time Curve

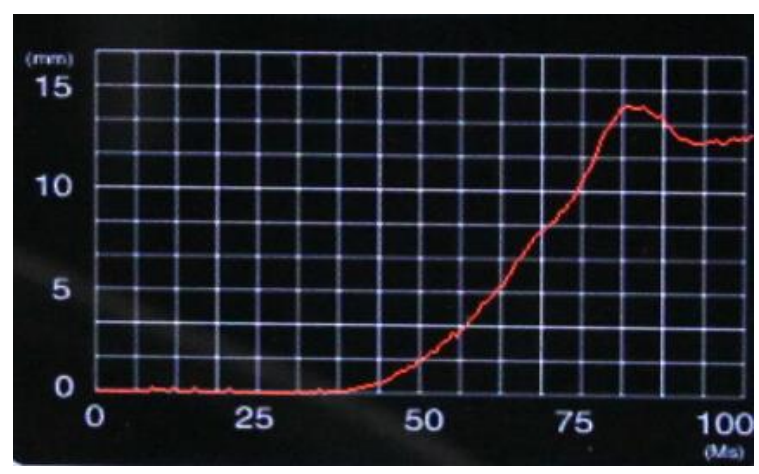

Fig. 7. Closing Displacement - Time Curve

As seen from the above tables and figures, the mechanical characteristics, the tripping, closing switching circuit of the circuit breaker is consistent with the measurement requirements. Moreover, it also truly reflects the actual work situation and effectively reaches its monitoring goals.

\section{ACKNOWLEDGMENT}

The project has been supported by the Innovation Program of Shanghai Municipal Education Commission "Vehicle Collision Avoidance System based on Vehicle Wireless Communication" (No.12YZ151).

\section{REFERENCES}

[1] XuGuo-zheng, et al. Principles and applications of high voltage circuit breakers [M].Beijing Tsinghua University Press, 2000.

[2] Lü Yi-hang, Li Jing, Dai Huai-zhi, et al.Research and Development of the On-line Synthetic Monitoring System of High Voltage Circuit Breakers[J]. Electric Power,2004,37(3) : 68-71.

[3] Ma Qiang, Li Kun, Rong Ming-zhe. Design of System on Chip for Switchgear On-line Monitoring [J]. Automation of Electric Power Systems, 2005, 29(3):73-76

[4] Dai Huai-zhi, Lü Yi-hang, Jia Shen-li, et al. Design of On-line Monitoring System for Circuit Breaker [J]. High Voltage Apparatus, 2004,40(2): 104-106

[5] H. H. Zeineldin, el al. High Voltage Circuit Breaker Modeling for Online Model-Based Monitoring and Diagnosis[J]. IEEE Transactions on Power Delivery, 2008, 3(4): 317-322.

[6] Xiong Xiao-fu, et al.Distributed monitoring system based on DSP and ARM for mechanical characteristic of high voltage [J]. Power System Protection and Control, 2009, 3(16)

[7] Liu Qiao, Miao Si-en, Implementation of the Communication between ARM and DSP Based on HPI[J]. Communications Electronics,2010, 47(3):52-54.

[8] Liu Chang, Tao Ran, Host-Port Interface (HPI) Application Based on DSP and ARM[J]. Dual-use technologies and products,2006,41-42 